\title{
The First Law for Slowly Evolving Horizons
}

\author{
Ivan Booth* \\ Department of Mathematics and Statistics, Memorial University of Newfoundland, \\ St. John's, Newfoundland and Labrador, Canada AlC $5 S 7$ \\ Stephen Fairhurst ${ }^{\dagger}$ \\ Theoretical Physics Institute, Department of Physics, University of Alberta, Edmonton, Alberta, Canada T6G $2 J 1$
} (Received 5 August 2003; published 6 January 2004)

\begin{abstract}
We study the mechanics of Hayward's trapping horizons, taking isolated horizons as equilibrium states. Zeroth and second laws of dynamic horizon mechanics come from the isolated and trapping horizon formalisms, respectively. We derive a dynamical first law by introducing a new perturbative formulation for dynamic horizons in which "slowly evolving" trapping horizons may be viewed as perturbatively nonisolated.
\end{abstract}

DOI: 10.1103/PhysRevLett.92.011102

The laws of black hole mechanics are one of the most remarkable results to emerge from classical general relativity. Recently, they have been generalized to locally defined isolated horizons [1]. However, since no matter or radiation can cross an isolated horizon, the first and second laws cannot be treated in full generality. Instead, the first law arises as a relation on the phase space of isolated horizons rather than as a truly dynamical relationship. Even existing physical process versions of the first law $[2,3]$ consider transitions between infinitesimally separated isolated (or Killing) horizons. In this Letter, we introduce a framework which allows us to extend the first law to all slowly evolving horizons, even those whose initial and final states are not infinitesimally separated. Additionally, we provide a simple characterization of how close a horizon is to equilibrium, which will be useful in the final stages of numerical simulations of black hole collisions. To obtain such a law, we first need a local dynamical definition of a black hole horizon. Hayward has introduced future outer trapping horizons (FOTHs) for exactly this purpose. Furthermore, he has shown that they necessarily satisfy the second law - their area cannot decrease in time [4].

In this Letter, we will show that both the zeroth and first laws are also applicable to FOTHs. In order to do so, we introduce dynamical notions of surface gravity and angular momentum which are applicable to all such horizons. It follows immediately that the surface gravity is necessarily constant if the horizon is in equilibrium (i.e., isolated). Next, we introduce the notion of a slowly evolving horizon, for which the gravitational and matter fields are slowly changing. It is only in this limited context that we expect to obtain the dynamical first law. We will show that this is indeed the case.

Let us begin by recalling Hayward's definition.

Future outer trapping horizon.-A future outer trapping horizon (FOTH) is a smooth three-dimensional submanifold $H$ of space-time which is foliated by a preferred family of spacelike two-sphere cross sections
PACS numbers: 04.70.Bw, 04.25.Dm, 04.25.Nx

$H_{v}$, with future-directed null normals $\ell$ and $n$. The expansion $\theta_{(\ell)}$ of the null normal $\ell$ vanishes. Further, both the expansion $\theta_{(n)}$ of $n$ and $\mathcal{L}_{n} \theta_{(\ell)}$ are negative.

This definition captures the local conditions by which we would hope to distinguish a black hole. Specifically, the expansion of the outgoing light rays is zero on the horizon, positive outside, and negative inside. Additionally, the ingoing null rays are converging. As we will see, these horizons can be spacelike or null and include both equilibrium and nonequilibrium states - an important feature for our perturbative study. By comparison, if one is interested only in the dynamical phase, the spacelike dynamical horizons recently introduced by Ashtekar and Krishnan [5] are more relevant. However, such horizons are always expanding and thus not so suitable for studying transitions to and from equilibrium.

Hayward [4] has extensively studied the properties of FOTHs. Here, we summarize only those of his results which are important for our work. First, consider the quantities associated with the null vector fields $\ell$ and $n$, which we normalize so that $\ell \cdot n=-1$. We denote their relative expansions $\theta_{(\ell)}$ and $\theta_{(n)}$. Their twists are zero since they are normal to the $H_{v}$ cross sections of the horizon. Finally, we write their shears as $\sigma_{a b}^{(\ell)}$ and $\sigma_{a b}^{(n)}$.

Next, for each choice of the fields $\ell$ and $n$, there exists a scalar field $C$ on $H$ so that

$$
\mathcal{V}^{a}=\ell^{a}-C n^{a} \text { and } \tau_{a}=\ell_{a}+C n_{a}
$$

are, respectively, tangent and normal to the horizon. Note that $\mathcal{V} \cdot \mathcal{V}=-\tau \cdot \tau=2 C$. Hayward [4] has shown that, if the null energy condition holds, then

$$
C \geq 0
$$

on a FOTH. Thus, the horizon must be either spacelike or null, and the second law of trapping horizon mechanics follows quite easily. If $\tilde{q}_{a b}$ is the two metric on the cross sections, and $\sqrt{\tilde{q}}$ is the corresponding area element, then

$$
\mathcal{L}_{\mathcal{V}} \sqrt{\tilde{q}}=-C \theta_{(n)} \sqrt{\tilde{q}} .
$$


By definition, $\theta_{(n)}$ is negative and we have just seen that $C$ is non-negative. Hence, we obtain the local second law: If the null energy condition holds, then the area element $\sqrt{\tilde{q}}$ of a FOTH is nondecreasing along the horizon [4]. Clearly, on integration, the same law applies to the total area of the horizon. It is nondecreasing and remains constant if and only if the horizon is null.

To further restrict the rescaling freedom of the null vectors, we require that $\mathcal{L}_{\mathcal{V}} v=1$, where $v$ is the foliation parameter labeling the cross sections. Thus, choosing the foliation parameter fixes the length of $\mathcal{V}^{a}, \tau^{a}$, and the null vectors. However, at this stage, we are still free to change the foliation labeling and, by doing so, rescale $\mathcal{V}^{a}$ and the null vectors by functions of $v$. From the isolated horizon perspective, the normalization we have chosen is very natural, as in that case $\mathcal{V}^{a}=\ell^{a}$, and it is customary to choose the foliation parameter $v$, so that $n=-d v$.

Physical characterization of trapping horizons.-The laws of black hole mechanics are given in terms of physical quantities such as energy, angular momentum, surface gravity, and area. One of the advances of the isolated horizon formalism was to provide definitions of all these quantities at the horizon, without reference to spacelike infinity or the space-time in which the horizon is embedded [1,2]. In generalizing these definitions to FOTHs, we will be motivated by two requirements: (i) the new definitions should match the old ones when trapping horizons are isolated and (ii) the new definitions should depend only on quantities that are intrinsic to the horizon, and as such truly be properties of the horizon itself. That said, the ultimate justification for these expressions will be found in their utility in the calculations that follow.

For isolated horizons, both surface gravity and angular momentum were defined with respect to a one-form $\omega_{a}$ which can be written as

$$
\omega_{a}:=-n_{b} \underset{\leftarrow a}{\nabla} \ell^{b},
$$

where the arrow signifies that the derivative is pulled back to the horizon. Then, the isolated horizon surface gravity is given by $\kappa_{\ell}=\ell^{a} \omega_{a}$ while angular momentum information is contained in the other components of $\omega_{a}$.

Now, $\omega_{a}$ written in this form [Eq. (4)] continues to be an intrinsically well-defined quantity for trapping horizons. Then, an obvious generalization is to define

$$
\begin{aligned}
\kappa_{v} & :=\mathcal{V}^{a} \omega_{a}\left(=-n_{b} \mathcal{V}^{a} \nabla_{a} \mathcal{V}^{b}\right) \quad \text { and } \\
J_{\varphi} & :=\frac{1}{8 \pi} \int_{H_{v}} d^{2} x \sqrt{\tilde{q}} \varphi^{a} \tilde{\boldsymbol{\omega}}_{a},
\end{aligned}
$$

where $\varphi^{a}$ is any vector field tangent to the horizon cross sections, and $\tilde{\omega}_{a}$ is the projection of $\omega_{a}$ into the twosurface $H_{v}$. In the case where $\varphi^{a}$ is divergence free, $J_{\varphi}$ is independent of the normalization of $\ell$ and equal to other popular expressions for angular momentum (such as the Komar, Brown-York [6], or Ashtekar-Krishnan [5] definitions). It is clear that both the surface gravity $\kappa_{v}$ and angular momentum $J_{\varphi}$ expressions reduce to their isolated horizon values if the horizon is null.

The constraint law.-Consider the set of vector fields in $H$ that generate one-parameter families of diffeomorphisms that map two-dimensional cross sections $H_{v}$ of the horizon into each other. Any such vector field may be written in the form $X^{a}=x_{0} \mathcal{V}^{a}+\tilde{x}^{a}$, for some function $x_{0}(v)$ and vector field $\tilde{x}^{a}$ that is everywhere tangent to an appropriate cross section. Then, by integrating the $\tau^{a} X^{b}$ component of the Einstein equations over $H_{v}$, we obtain the following relationship:

$$
\begin{aligned}
\frac{1}{8 \pi G} \int_{H_{v}} d^{2} x\left[\kappa_{v} \mathcal{L}_{X} \sqrt{\tilde{q}}+\tilde{x}^{a} \mathcal{L}_{\mathcal{V}}\left(\sqrt{\tilde{q}} \tilde{\omega}_{a}\right)\right]= & \int_{H_{v}} d^{2} x \sqrt{\tilde{q}}\left[T_{a b} X^{a} \tau^{b}\right]+\frac{1}{16 \pi G} \int_{H_{v}} d^{2} x \sqrt{\tilde{q}}\left(\sigma^{(\ell) a b}+C \sigma^{(n) a b}\right)\left(\mathcal{L}_{X} \tilde{q}_{a b}\right) \\
& +\frac{1}{16 \pi G} \int_{H_{v}} d^{2} x\left[2 C \sqrt{\tilde{q}}\left(\mathcal{L}_{X} \theta_{(n)}\right)+C \theta_{(n)}\left(\mathcal{L}_{X} \sqrt{\tilde{q}}\right)\right] .
\end{aligned}
$$

A full derivation of this result will be given in [7], and in another paper we will see that this relation plays a crucial role in the Hamiltonian formulation of general relativity on manifolds with FOTHs as boundaries [8]. Here, we show that in certain restricted situations Eq. (6) becomes a first law for dynamical black holes.

Quasistationary horizons.-At first glance, one might think that (6) is already the dynamical first law of black hole mechanics. After all, it relates rates of change of area and angular momentum to fluxes across the horizon. However, there are several reasons why this is not so. The first and most important is that we should not expect the standard $\kappa \dot{a}$ form of the first law to hold in all dynamical situations. In thermodynamics, it is only in the quasistatic case that it is possible to write the energy balance equation as

$$
d E=T d S+\text { work terms. }
$$

Furthermore, in the general case, there is no clear interpretation of the right-hand side of (6) as a flux of energy through the horizon. Instead, we will require the horizon to be "quasistationary" and then obtain a first law.

Heuristically, it is clear that the properties of a quasistationary horizon, such as the area and surface gravity, should be slowly varying. However, since there is a rescaling freedom in the vector field $\mathcal{V}$, requiring $\left(\mathcal{L}_{\mathcal{V}} \sqrt{\tilde{q}}\right) / \sqrt{\tilde{q}}$ to be small is not a meaningful condition-it can be satisfied on any trapping horizon by suitably rescaling $\mathcal{V}$. Furthermore, we cannot fix the norm of $\mathcal{V}$ (or its average) to unity since we are interested in the limit as $\mathcal{V}$ becomes null. Instead, we introduce the 
one-form $\chi_{a}:=d_{a} v$ satisfying $\mathcal{V} \cdot \chi=1$, and require $\left(\mathcal{L}_{\mathcal{V}} \sqrt{\tilde{q}}\right)\left(\mathcal{L}_{\chi} \sqrt{\tilde{q}}\right) / \tilde{q}$ to be small compared to the characteristic scale of the horizon. This condition is invariant under rescalings, and the expression vanishes whenever the horizon is null. On a spacelike horizon, $\left(\mathcal{L}_{\mathcal{V}} \sqrt{\tilde{q}}\right)\left(\mathcal{L}_{\chi} \sqrt{\tilde{q}}\right)=\tilde{q} C \theta_{(n)}^{2}$ and so is closely related to the expansion of the surface [see Eq. (3)]. By evaluating this expression, we can invariantly determine the expansion rate of the horizon and choose the foliation accordingly. Then, we require other fields to be slowly varying with respect to this foliation.

Slowly evolving horizon.-A region of a future outer trapping horizon $H$ with $v \in\left[v_{1}, v_{2}\right]$ is slowly evolving (at a rate $\epsilon$ ) if there exists a parameter $\epsilon \ll 1$ such that (i) on every cross section $H_{v}$ with $v \in\left[v_{1}, v_{2}\right]$,

$$
\int_{H_{v}} d^{2} x \sqrt{\tilde{q}}\left(\tilde{q}^{a b} \nabla_{a} \mathcal{V}_{b}\right)\left(\tilde{q}^{c d} \nabla_{c} \chi_{d}\right) \leq \epsilon^{2} .
$$

(ii) The foliation parameter $v$ is chosen so that $|\mathcal{V}|=$ $\sqrt{2 C} \sim \epsilon$, and its rates of change are similarly small over the horizon. (iii) The one-form $\tilde{\boldsymbol{\omega}}_{a}$ and expansion $\theta_{(n)}$ are slowly evolving: $\left|\mathcal{L}_{\mathcal{V}} \tilde{\boldsymbol{\omega}}_{a}\right| \leq \epsilon / r_{H}^{2}$ and $\left|\mathcal{L}_{\mathcal{V}} \theta_{(n)}\right| \leq \epsilon / r_{H}^{2}$, where $r_{H}$ is the area radius of the horizon, $a_{H}=4 \pi r_{H}^{2}$. (iv) $|\tilde{\mathcal{R}}|,|\omega|^{2},\left|\sigma^{(n)}\right|^{2}$, and $T_{a b} n^{a} n^{b} \sim 1 / r_{H}^{2}$ or smaller.

The first condition gives an invariant characterization of slow expansion. The second condition restricts the foliation to guarantee that the area is slowly evolving with respect to $\mathcal{V}^{a}$, specifically $\left(\mathcal{L}_{\mathcal{V}} r_{H}\right) \sim \epsilon^{2}$. The third requires other geometric quantities to also be slowly evolving, while the fourth fixes a reasonable horizon geometry and demands that conditions in the surrounding space-time not be too extreme; here $\tilde{\mathcal{R}}$ is the Ricci scalar of the two surfaces $H_{v}$. An immediate consequence of our definition is that isolated horizons are examples of slowly evolving horizons with $\epsilon=C=0$ [provided condition (iv) is satisfied].

Let us now consider the implications of this definition. Here, we will simply state the consequences; a more complete discussion will be given in [7]. For concreteness, we restrict the allowed matter to be scalar and/or electromagnetic fields. From a projection of the Einstein equations and condition 2 , we can show that

$$
\left|\sigma_{a b}^{(\ell)}\right| \sim \epsilon \text { and } T_{a b} \ell^{a} \ell^{b} \sim \epsilon^{2} .
$$

(Here and in the following, we choose to focus on the $\epsilon$ factors. The powers of $r_{H}$ required to make the equations dimensionally correct are omitted.) Then, the allowed form of the matter fields forces $T_{a b} \ell^{a} \tilde{x}^{b} \sim \epsilon$ and $\mathcal{L}_{\mathcal{V}}\left(T_{a b} \ell^{a} n^{b}\right) \sim \epsilon$. Further application of the Einstein equations and Bianchi identities gives the Weyl components $\Psi_{0}, \Psi_{1} \sim \epsilon$ and $\mathcal{L}_{\mathcal{V}} \tilde{\mathcal{R}} \sim \epsilon$. On an isolated horizon, each of these quantities would be zero. Lastly, condition (iii) is sufficient to guarantee that

$$
\left|\mathcal{L}_{\mathcal{V}} \kappa_{v}\right| \sim \epsilon \text { and }\left|\tilde{q}_{a}^{b} \nabla_{b} \kappa_{v}\right| \sim \epsilon .
$$

Therefore, on a slowly evolving horizon, the surface gravity is a slowly varying function and we can write

$$
\kappa_{v}=\kappa^{(0)}+\epsilon \kappa^{(1)},
$$

where $\kappa^{(0)}$ is a constant. Recall that an isolated horizon corresponds to $\epsilon=0$. Hence, we immediately obtain the zeroth law: The surface gravity of a FOTH is constant if the horizon is isolated.

The dynamical first law.-We can now derive the first law for slowly evolving horizons. First, we consider evolution with respect to $\mathcal{V}^{a}$, an appropriate evolution vector for a horizon with no obvious axis of symmetry-a nonrotating horizon. Setting $\tilde{x}^{a}=0$ and $x_{0}=1$ in the constraint law (6) and expanding in powers of $\epsilon$, we find that all terms vanish at zeroth and first order. Then, to $\mathcal{O}\left(\epsilon^{2}\right)$, the first law for a slowly evolving horizon reads

$$
\begin{aligned}
\frac{1}{8 \pi G} \kappa^{(0)} \dot{a}_{H} & =\dot{E} \\
& :=\int_{H_{v}} d^{2} x \sqrt{\tilde{q}}\left[T_{a b} \ell^{a} \ell^{b}+\frac{1}{8 \pi G}\left|\sigma^{(\ell)}\right|^{2}\right],
\end{aligned}
$$

where the dot signifies a derivative with respect to $v$. It says that the surface gravity multiplied by the change in area is equal to a flux of energy through the horizon. This flux is comprised of two terms, both of which are positive. The first is the flux of matter through the horizon and is familiar from standard physical process versions of the first law [3]. The second term is a flux of gravitational shear through the horizon-which would naturally be interpreted as a flux of gravitational radiation. A similar term has been obtained previously by Hawking [9] when considering perturbations of an event horizon.

We would like to "integrate" (11) in order to obtain an expression for the energy of the horizon. To this end, we further restrict the choice of $\mathcal{V}^{a}$ by requiring that

$$
\kappa^{(0)}=\frac{1}{2 r_{H}} .
$$

Note that while we cannot prove that this rescaling is possible on every slowly evolving horizon, we can show that, if the horizon satisfies a certain genericity condition, it will be. In particular, perturbations to Schwarzschild and nonextremal Kerr horizons will satisfy the condition. Since $\kappa^{(0)}$ is a function of $r_{H}$ alone, we can integrate the $\kappa \dot{a}$ term in (11) to obtain the usual expression for the energy

$$
E=\frac{r_{H}}{2 G} .
$$

Rotating horizons.-Next, consider rotating horizons. In (5), we gave a definition for the angular momentum. However, this quantity only has a physical significance if the vector field $\varphi^{a}$ is an (approximate) symmetry of the horizon. Hence, we define the following.

Approximately symmetric horizon.-The vector field $\varphi^{a}$ is an approximate symmetry of a trapping horizon if 
(i) $\varphi^{a}$ is tangent to the cross sections of the horizon, generates a family of closed integral curves, and is normalized so that those curves have affine parameter length $2 \pi$. (ii) $\varphi^{a}$ is Lie dragged up the horizon by $\mathcal{V}^{a}$, i.e., $\mathcal{L}_{\mathcal{V}} \varphi^{a}=0$. (iii) $\varphi^{a}$ is an approximate symmetry of the horizon geometry and matter fields: $\left|\tilde{q}^{a b} \nabla_{a} \varphi_{b}\right|<\epsilon^{2}$, $\left|\mathcal{L}_{\varphi} \tilde{q}_{a b}\right|<\epsilon,\left|\mathcal{L}_{\varphi} \tilde{\omega}_{a}\right|<\epsilon,\left|\mathcal{L}_{\varphi} \theta_{(n)}\right|<\epsilon$, and $\left|T_{a b} \varphi^{a} \ell^{b}\right|<$ $\epsilon^{2}$. (These conditions are analogous to those satisfied by $\mathcal{V}^{a}$ on a slowly evolving horizon.)

For a slowly evolving horizon which is approximately symmetric, the angular momentum $J_{\varphi}$ is a meaningful quantity. Going back to the constraint law and setting $x_{0}=0$ and $\tilde{x}^{a}=\varphi^{a}$, we find its time rate of change. As in the nonrotating case, Eq. (6) will vanish at zeroth and first orders in $\epsilon$. To second order, we have

$$
\mathcal{L}_{\mathcal{V}} J_{\varphi}=\int_{H_{v}} d^{2} x \sqrt{\tilde{q}}\left[T_{a b} \tau^{a} \varphi^{b}+\frac{1}{16 \pi G} \sigma^{(\ell) a b}\left(\mathcal{L}_{\varphi} \tilde{q}_{a b}\right)\right] .
$$

Thus, the rate of change of angular momentum of the horizon depends upon the flux of matter and gravitational fields through the horizon.

To get a general first law, we would like to combine the rate of change of angular momentum with the first law for nonrotating horizons (11). To do this, we again fix the average value of the surface gravity as well as an angular velocity $\Omega$ (we are following a similar strategy to that taken in [5], though with the caveat that here only the average value of the surface gravity is fixed). We do this by requiring that both take the same values as in a Kerr space-time with the same $J_{\varphi}$ and $a_{H}$ :

$$
\Omega:=\frac{2 G J_{\varphi}}{r_{H} \sqrt{r_{H}^{4}+4 G^{2} J_{\varphi}^{2}}} \quad \text { and } \quad \kappa^{(0)}=\frac{r_{H}^{4}-4 G^{2} J_{\varphi}^{2}}{2 r_{H}^{3} \sqrt{r_{H}^{4}+4 G^{2} J_{\varphi}^{2}}} \text {. }
$$

With area and angular momentum slowly changing up the horizon, the angular velocity is also slowly varying.

Finally, we consider the full constraint law (6) with the evolution vector field $X^{a}$ chosen as

$$
t^{a}:=\mathcal{V}^{a}+\Omega \varphi^{a} .
$$

Then, we can once again expand out the constraint law (6) to order $\epsilon^{2}$ and this time obtain

$$
\begin{aligned}
\dot{E} & =\frac{1}{8 \pi G} \kappa^{(0)} \dot{a}+\Omega \dot{J}_{\varphi}, \quad \text { where } \\
\dot{E} & :=\int_{H_{v}} d^{2} x \sqrt{\tilde{q}}\left[T_{a b} t^{a} \tau^{b}+\frac{1}{16 \pi G} \sigma^{(\ell) a b}\left(\mathcal{L}_{t} \tilde{q}_{a b}\right)\right] .
\end{aligned}
$$

Since $\kappa^{(0)}$ and $\Omega$ are specific functions of only $r_{H}$ and $J_{\varphi}$, it is once again possible to integrate the left-hand side of the equation to obtain as an expression for the energy

$$
E=\frac{\sqrt{r_{H}^{4}+4 G^{2} J_{\varphi}^{2}}}{2 G r_{H}}
$$

This $E$ is equal to the energy of a rotating isolated horizon with the preferred choice of normalization for $\ell$, and, in particular, is the mass of a Kerr black hole with parameters $a_{H}$ and $J_{\varphi}$.

The notion of a slowly evolving horizon provides a bridge between the equilibrium of isolated horizons and the fully dynamical horizons of [5]. In particular, our first law (17) bears a striking resemblance to the dynamical horizon energy balance formula. There are, however, several important differences. First, we restrict to slowly evolving horizons, which are near to equilibrium. Doing so enables us to provide a locally defined surface gravity $\kappa_{v}$ which is shown to be slowly varying over the horizon. This surface gravity maintains its usual interpretation as the acceleration of a vector field, $\mathcal{V}^{a}$, along the horizon. Second, we do not obtain an equivalent of the $|\zeta|^{2}$ term of [5], which is likely related to angular momentum. However, we expect that term would vanish at the order of perturbation theory which we are considering. In the future, we plan to examine the connection between these two formulations in more detail and determine in what precise sense our first law can be seen as a perturbative form of the dynamical horizon energy balance formula.

In summary, we have examined conditions for which a slowly evolving FOTH may be said to be "perturbatively nonisolated." This has allowed us to obtain a truly dynamical version of the first law of black hole mechanics. However, we expect that the notion of a slowly evolving horizon will also find application in numerical studies of how horizons settle down to equilibrium. Specifically, one could track the approach of the parameter $\epsilon$ to zero.

We thank A. Ashtekar, C. Beetle, and B. Krishnan for discussions. S. F. was supported by the Killam Trusts at the University of Alberta and NSF Grant No. PHY0200852 at UWM; I. B. was supported by NSERC.

*Electronic address: ibooth@math.mun.ca

${ }^{\dagger}$ Electronic address: sfairhur@gravity.phys.uwm.edu

[1] A. Ashtekar, C. Beetle, and J. Lewandowski, Phys. Rev. D 64, 044016 (2001); A. Ashtekar et al., Phys. Rev. Lett. 85, 3564 (2000); A. Ashtekar, S. Fairhurst, and B. Krishnan, Phys. Rev. D 62, 104025 (2000).

[2] A. Ashtekar, C. Beetle, and S. Fairhurst, Classical Quantum Gravity 17, 253 (2000).

[3] S. Gao and R. M. Wald, Phys. Rev. D 64, 084020 (2001).

[4] S. A. Hayward, Phys. Rev. D 49, 6467 (1994).

[5] A. Ashtekar and B. Krishnan, Phys. Rev. Lett. 89, 261101 (2002); Phys. Rev. D 68, 104030 (2003).

[6] J. D. Brown and J.W. York, Phys. Rev. D 47, 1407 (1993).

[7] I. Booth and S. Fairhurst (to be published).

[8] I. Booth and S. Fairhurst (to be published).

[9] S. Hawking, The Event Horizon, in Black Holes, edited by B. deWitt and C. deWitt (Gordon and Breach, New York, 1973). 